\title{
Slowed Prosaccades and Increased Antisaccade Errors As a Potential Behavioral Biomarker of Multiple System Atrophy
}

\author{
Sarah H. Brooks ${ }^{1,2}$, Eliana M. Klier ${ }^{2}$, Stuart D. Red ${ }^{2}$, Neeti D. Mehta ${ }^{1}$, Saumil S. Patel ${ }^{2,3}$, \\ Alice Z. Chuang ${ }^{4}$, Jessika Suescun ${ }^{5}$, Mya C. Schiess ${ }^{5}$ and Anne B. Sereno ${ }^{2 *}$ \\ ${ }^{1}$ Department of Cognitive Sciences, Rice University, Houston, TX, United States, ${ }^{2}$ Department of Neurobiology and \\ Anatomy, McGovern Medical School, The University of Texas Health Science Center at Houston, Houston, TX, \\ United States, ${ }^{3}$ Department of Neuroscience, Baylor College of Medicine, Houston, TX, United States, ${ }^{4}$ Department of \\ Ophthalmology, McGovern Medical School, The University of Texas Health Science Center at Houston, Houston, TX, \\ United States, ${ }^{5}$ Department of Neurology, McGovern Medical School, The University of Texas Health Science Center at \\ Houston, Houston, TX, United States
}

Current clinical diagnostic tools are limited in their ability to accurately differentiate idiopathic Parkinson's disease (PD) from multiple system atrophy (MSA) and other parkinsonian disorders early in the disease course, but eye movements may stand as

OPEN ACCESS

Edited by:

Giuseppe De Michele, University of Naples Federico II, Italy

Reviewed by: Yasuo Terao, University of Tokyo, Japan Silmar Teixeira,

Federal University of Piauí, Brazil

*Correspondence: Anne B. Sereno anne.b.sereno@uth.tmc.edu

Specialty section: This article was submitted to Movement Disorders, a section of the journal

Frontiers in Neurology

Received: 13 March 2017 Accepted: 24 May 2017 Published: 20 June 2017

Citation: Brooks SH, Klier EM, Red SD, Mehta ND, Patel SS, Chuang AZ,

Suescun J, Schiess MC and

Sereno $A B$ (2017) Slowed

Prosaccades and Increased Antisaccade Errors As a Potential Behavioral Biomarker of Multiple System Atrophy.

Front. Neurol. 8:261. doi: 10.3389/fneur.2017.00261 objective and sensitive markers of disease differentiation and progression. To assess the use of eye movement performance for uniquely characterizing PD and MSA, subjects diagnosed with PD ( $N=21)$, MSA ( $N=11)$, and age-matched controls $(C, N=20)$ were tested on the prosaccade and antisaccade tasks using an infrared eye tracker. Twenty of these subjects were retested $\sim 7$ months later. Saccade latencies, error rates, and longitudinal changes in saccade latencies were measured. Both PD and MSA patients had greater antisaccade error rates than $\mathrm{C}$ subjects, but MSA patients exhibited longer prosaccade latencies than both $\mathrm{PD}$ and $\mathrm{C}$ patients. With repeated testing, antisaccade latencies improved over time, with benefits in $\mathrm{C}$ and PD but not MSA patients. In the prosaccade task, the normal latencies of the PD group show that basic sensorimotor oculomotor function remain intact in mid-stage PD, whereas the impaired latencies of the MSA group suggest additional degeneration earlier in the disease course. Changes in antisaccade latency appeared most sensitive to differences between MSA and PD across short time intervals. Therefore, in these mid-stage patients, increased antisaccade errors combined with slowed prosaccade latencies might serve as a useful marker for early differentiation between PD and MSA, and, antisaccade performance, a measure of MSA progression. Together, our findings suggest that eye movements are promising biomarkers for early differentiation and progression of parkinsonian disorders.

Keywords: saccade, eye movement, Parkinson's disease, multiple system atrophy, latency, error rate

\section{INTRODUCTION}

Parkinsonian disorders refer to a group of diseases linked to basal ganglia dopamine insufficiency. The most common form is idiopathic Parkinson's disease (PD) and the remaining syndromes are grouped as atypical Parkinsonism (AP) (1). AP encompasses a less prevalent group of disorders including multiple system atrophy (MSA), progressive supranuclear palsy (PSP), Lewy body dementia 
(LBD), and corticobasal degeneration (CBD), among others (2). Parkinsonian disorders share clinical features including the presence of bradykinesia plus one of the following: muscular rigidity, resting tremor, or postural instability; however, they differ in pathophysiology and progression rate $(1,3)$. As novel neuroprotective therapies are developed, it becomes increasingly crucial for earlier and more accurate diagnosis.

Specifically, due to early overlapping symptoms, PD and MSA pose challenges in differential diagnosis, critical for early disease prognosis. Currently, no biomarkers can differentiate between the etiologies and clinical measures lack sensitivity and objectivity to uniquely characterize early stages of $\operatorname{PD}$ and $\operatorname{MSA}(4,5)$. Therefore, biomarkers are needed to model the early differences and progression of PD and MSA.

Note that MSA is characterized by glial cytoplasmatic inclusions in different regions including the putamen, caudate nucleus, substantia nigra, locus ceruleus, pontine nuclei, inferior olivary nucleus, Purkinje cell layer of the cerebellum, and intermediolateral cell columns (6), and the degree of neuronal loss in these areas determines the clinical presentation that phenotypically presents as two variants: parkinsonian (MSA-P) and cerebellar (MSA-C). The MSA-P subtype is associated with predominantly nigrostriatal degeneration and represents between 68 and $82 \%$ of the patients in the western hemisphere, whereas the MSA-C variant is associated with primarily olivopontocerebellar atrophy and is predominant in Japan (7). These categories refer to dominant motor features that can change over time, hence changing the designation of the variant. Thus, these subtypes represent the main characteristics at the time the patient is evaluated but are subject to variation over disease progression (8), mainly based on the widely distributed and rapidly progressive pathological burden. In our study population, the two subtypes are present in nearly equal numbers (55\% MSA-P and 45\% MSA-C).

Eye movements are a highly sensitive and objective measures used to test various levels of nervous system function, including cognitive status, making them a suitable measure for modeling PD and MSA disease course (9). Particularly, evidence suggests that saccadic performance may be useful in characterizing Parkinsonian disorders (10-13). Two paradigms, the prosaccade and antisaccade tasks, are disrupted by lesions in frontal and midbrain regions (14-17), which are affected by the pathology characteristic of Parkinsonian disorders. Prosaccades rely on intact sensorimotor and reflexive motor systems including midbrain regions, whereas antisaccades require additional voluntary control to suppress the reflexive response and to program and initiate the willful response [processes involving the frontal lobe (18-21)]. With known frontal lobe deficits, the antisaccade task is a powerful measure of cognitive deficit in neurodegenerative diseases (22-26).

Studies attempting to characterize the eye movements of parkinsonian disorders $(22,27,28)$ have inconsistent results regarding which abnormalities are shared and which are specific to particular disorders. Further, there is even disagreement about whether prosaccade latencies in $\mathrm{PD}$ are slower, faster, or indistinguishable $(29,30)$. There are many variables that have been shown to affect saccade parameters including age (31), dopaminergic medication (24), and disease stage $(30,32)$. Previous work suggests similar progression of eye movement abnormalities in PD and MSA-P patients with similar disease severity tested $4 \mathrm{~h}$ after intake of L-DOPA (30). Here, we studied patients with established diagnoses in the conventionally defined medication "off" state, aiming to characterize eye movements of mid-stage PD and MSA, to identify differences in performance that could serve as potential markers for early differential diagnosis. We examined latencies and error rates of control, PD, and MSA subjects on reflexive and voluntary tasks at a single time point, and also tracked performances across time by testing at a second time point $\sim 7$ months later. With combined single time point and longitudinal data, we aimed to identify measures that already differ in mid-stage that may be promising for differentiating PD and MSA in early stages, and to characterize measures that are useful for tracking disease progression and/or the effects of interventions.

\section{MATERIALS AND METHODS}

\section{Subjects}

Patients with PD $(N=21)$, MSA $(N=11$; MSA-C $=5$ and MSA-P $=6)$, and controls $(N=20)$ were recruited from the UTHealth Movement Disorders Clinic (Table 1). Subjects provided written informed consent in accordance with the Declaration of Helsinki and were enrolled into a study approved by the Institutional Review Board at the University of Texas Health Science Center at Houston. At each visit, with the exception of nine controls and one PD participant at a second session, participants were tested on the Unified Parkinson's Disease Rating Scale (UPDRS) and the Hoehn and Yahr (H\&Y). At their initial visit, with the exception of nine controls and two MSA participants at a second session, participants were tested on the Montreal Cognitive Assessment and University of Pennsylvania Smell Identification Tests. PD diagnosis was based on the United Kingdom Brain Bank criteria (4). Additionally, PD diagnosis required a robust and sustained response to levodopa or dopamine agonist therapy, defined as $>30 \%$ reduction in symptoms on the UPDRS (33). The second consensus statement by the American Autonomic Society and American Academy of Neurology was used for MSA diagnosis (8). Subjects with Parkinsonian symptoms due to PSP, LBD, CBD, vascular Parkinsonism, or medicine-/toxin-induced Parkinsonism were excluded. The diagnosis was confirmed by a movement disorders specialist and, to exclude advanced disease and establish mid-stage disease, we used an H\&Y disability scale (34) with a cutoff of $\leq 3.5$ in the conventionally defined "off" medication state.

All 52 participants were tested at time point 1 (T1) on the eye movement tasks, and 20 returned at a second time point (T2) on average 7.4 months later (range 4.6-13.8). Demographics were similar among the three groups $[F(2,49)=1.84, p=0.17$ for age; Fisher's exact test, $p=0.66$ for gender and $p=0.20$ for race/ethnicity]. However, clinical characteristics were significantly different among groups (Table 1). In addition, the subset (9 PDs, 6 MSAs, 5 controls) retested at T2 was not different in both demographics and clinical characteristics from those that were not retested, $F(1,50)=0.09, p=0.77$ for age; Fisher's exact test, $p=0.39$ for gender, and $p=0.36$ for race/ethnicity; 
TABLE 1 | Participant demographics of control (C), Parkinson's disease (PD), and multiple system atrophy (MSA) groups tested at time point 1 (T1) and time point 2 (T2).

\begin{tabular}{|c|c|c|c|c|c|}
\hline & & $C(N=20)$ & PD $(N=21)$ & MSA $(N=11)$ & Test statistics $\left[p^{\mathrm{a}}\right]$ \\
\hline \multirow[t]{9}{*}{ All participants (T1) } & Age, mean $( \pm S D)$ [range] & $57.6( \pm 10.5)[41-80]$ & $63.4( \pm 10.0)[45-78]$ & $62.3( \pm 9.4)[39-74]$ & $F(2,49)=1.84[p=0.17]$ \\
\hline & Gender, No. female (\%) & $9(45 \%)$ & $8(38 \%)$ & $3(27 \%)$ & Fischer $=0.025[p=0.66]$ \\
\hline & H\&Y, mean ( $\pm S D)$ [range] & $0.0( \pm 0.0)^{\mathrm{b}}[0.0-0.0]$ & $1.7( \pm 0.7)[1.0-3.5]$ & $3.1( \pm 1.0)[1.5-5.0]$ & $F(2,40)=50.12[p<0.001]$ \\
\hline & UPDRS-T, mean ( \pm SD) [range] & $2.6( \pm 3.0)^{\mathrm{b}}[0-9]$ & $36.5( \pm 16.3)[12-75]$ & $52.8( \pm 15.5)[29-91]$ & $F(2,40)=37.47[p<0.001]$ \\
\hline & UPDRS-M, mean ( \pm SD) [range] & $0.6( \pm 1.3)^{b}[0-4]$ & $22.1( \pm 10.4)[7-43]$ & $29.5( \pm 9.7)[15-47]$ & $F(2,40)=33.21[p<0.001]$ \\
\hline & MoCA, mean ( \pm SD) [range] & $28.6( \pm 1.6)^{b}[26-30]$ & $27.1( \pm 3.2)[18-30]$ & $24.6( \pm 4.2)[16-29]$ & $F(2,40)=4.50[p=0.017]$ \\
\hline & UPSIT, mean ( \pm SD) [range] & $37.3( \pm 1.2)^{\mathrm{b}}[35-39]$ & $22.4( \pm 6.6)[12-29]$ & $29.7( \pm 6.7)^{c}[13-38]$ & $F(2,39)=24.48[p<0.001]$ \\
\hline & $\begin{array}{l}\text { Duration of disease in years, } \\
\text { mean }( \pm S D)\end{array}$ & $\mathrm{n} / \mathrm{a}$ & $6.9( \pm 3.2)[2-14]$ & $5.2( \pm 1.8)[3.5-10]$ & $F(1,30)=2.53[p=0.12]$ \\
\hline & & $(N=5)$ & $(N=9)$ & $(N=6)$ & \\
\hline \multirow{5}{*}{$\begin{array}{l}\text { Repeat tested } \\
\text { participants (T2) }\end{array}$} & Age, mean $( \pm S D)$ [range] & $60.3( \pm 11.0)[42-72]$ & $62.1( \pm 12.2)[45-78]$ & $62.3( \pm 12.2)[40-75]$ & $F(2,17)=0.05[p=0.95]$ \\
\hline & Gender, No. female (\%) & $2(40 \%)$ & $3(33 \%)$ & $1(17 \%)$ & Fischer $=0.13[p=0.70]$ \\
\hline & H\&Y, mean ( \pm SD) [range] & $0.0( \pm 0.0)[0.0-0.0]$ & $1.6( \pm 0.6)[1.0-2.5]$ & $2.8( \pm 0.5)[2.0-3.5]$ & $F(2,17)=46.72[p<0.001]$ \\
\hline & UPDRS-T, mean ( \pm SD) [range] & $4.4( \pm 5.7)[0-12]$ & $33.8( \pm 13.8)^{\mathrm{d}}[17-59]$ & $53.3( \pm 13.2)$ [33-69] & $F(2,16)=22.61[p<0.001]$ \\
\hline & UPDRS-M, mean ( \pm SD) [range] & $0.4( \pm 0.9)[0-2]$ & $23.3( \pm 8.9)[13-37]$ & $31.3( \pm 9.1)[22-46]$ & $F(2,17)=22.46[p<0.001]$ \\
\hline
\end{tabular}

H\&Y, Hoehn and Yahr; UPDRS-T, UPDRS Total; UPDRS-M, UPDRS Motor; MoCA, Montreal Cognitive Assessment; UPSIT, University of Pennsylvania Smell Identification Test. ${ }^{a} p$ Values (p) comparing among groups using one way analysis of variance (for gender: Fischer's exact test).

${ }^{b}$ Nine data points missing.

'One data point missing.

'One data point missing.

$F(1,41)=0.46, p=0.50$ for $\mathrm{H} \& \mathrm{Y} ; F(1,40)=0.07, p=0.79$ for UPDRS Total; and $F(1,41)=0.14, p=0.71$ for UPDRS Motor.

\section{Apparatus and Procedure}

Eye movements were recorded using an ISCAN RK-826-PCI infrared eye-tracking system. The spatial resolution was $\sim 0.5^{\circ}$ of visual angle, and temporal resolution was $4 \mathrm{~ms}(240 \mathrm{~Hz})$. Subjects were seated in front of a 17-inch CRT monitor with their heads stabilized on a chin rest positioned $72 \mathrm{~cm}$ from the screen. Before testing, the apparatus was calibrated by having the subject look to nine positions on the screen, arranged as a $3 \times 3$ matrix, indicated by $0.2^{\circ} \times 0.2^{\circ}$ white boxes on a black background. Participants then received instructions on the prosaccade and antisaccade tasks. A gray fixation point $\left(0.2^{\circ}\right.$ diameter $)$ was illuminated in the center of the black screen. Target stimuli were $0.2^{\circ} \times 0.2^{\circ}$ white squares that appeared in landmark boxes $\left(1.1^{\circ} \times 1.1^{\circ}\right) 7^{\circ}$ to the top, bottom, right, and left of the fixation point. Saccadic movements were defined by specific position and velocity criteria. For saccade initiation, eye velocity had to be $>47.5^{\circ} \mathrm{s}$, and for saccade termination, velocity had to be $<12^{\circ} \%$ s and within $4.4^{\circ}$ of the eye movement goal. PD and MSA participants were tested in the conventionally defined "off" state.

\section{Eye-Tracking Tasks}

For the prosaccade task, participants made an eye movement to the illuminated target (Figure 1A), whereas for the antisaccade task, participants made an eye movement to the mirror opposite location (Figure 1D). Each task contained 48 trials and was preceded by 8 practice trials. The trials were self-paced; once subjects fixated the center point for $400 \mathrm{~ms}$, the target appeared randomly in one of the four landmark boxes and remained illuminated until response. The fixation point was extinguished simultaneously with the target presentation and subjects looked as quickly as possible to the appropriate box (the target for prosaccade, opposite the target for antisaccade). Target position was balanced across the four possible target locations. For trials that timed out (subject failed to look to a landmark boxes $1492 \mathrm{~ms}$ after target onset) and for trials in which subjects made initial eye movements to the wrong box, subjects received visual ("wrong location") and auditory (low tone) feedback. Trials interrupted by blinks and timed out trials were aborted and randomly re-presented. Task order was counterbalanced across subjects.

\section{DATA ANALYSIS}

Due to technical problems for two MSA participants at T1, the antisaccade task was not performed. For all remaining data, saccade latencies $<100$ or $>900 \mathrm{~ms}$ were excluded as not targetrelated responses. This eliminated 1.6\% (C), 4.0\% (PD), and 5.1\% (MSA) of prosaccades and $2.9,4.7$, and $7.1 \%$ of antisaccades at $\mathrm{T} 1$, and $0.4,3.5$, and $3.3 \%$ of prosaccades and $2.9,3.7$, and $8.3 \%$ of antisaccades at $\mathrm{T} 2$.

\section{Direction Error Rate}

Direction error rate was calculated as the number of trials with initial eye movement responses to the wrong location divided by the total remaining number of trials. Direction error rate was 1.4 and $33.1 \%$ at $\mathrm{T} 1$ and 1.8 and $32.0 \%$ at $\mathrm{T} 2$, for prosaccade and antisaccade tasks, respectively.

\section{Saccade Latency}

Saccade latency was defined as the time from target onset to saccade initiation. After excluding direction error trials, the remaining correct trials were trimmed for outliers: trials outside 2.5 SDs of the subject's mean saccade latency for each task and time point were removed, resulting in the removal of an additional 3.0 and 
A

Prosaccade

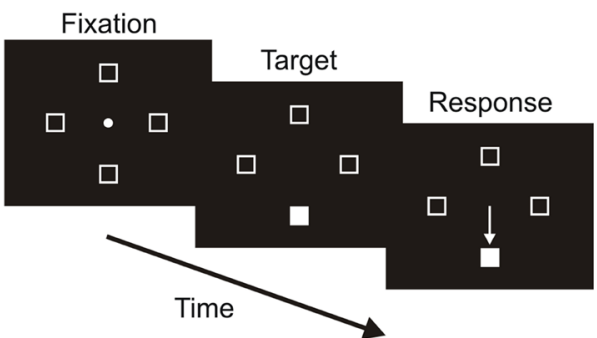

B

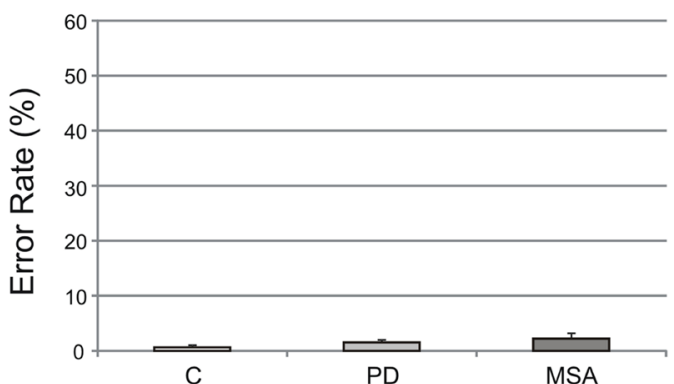

C

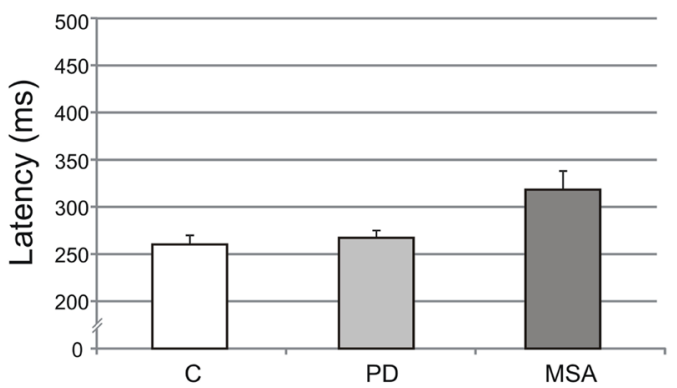

D

Antisaccade

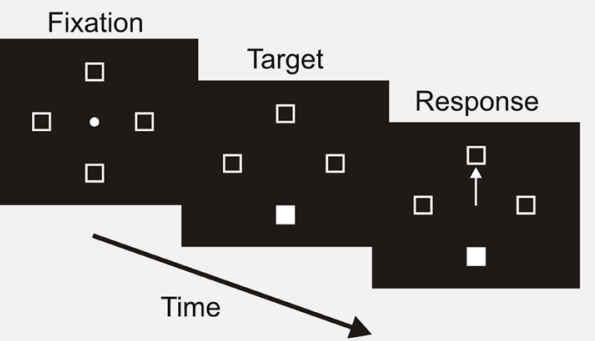

E

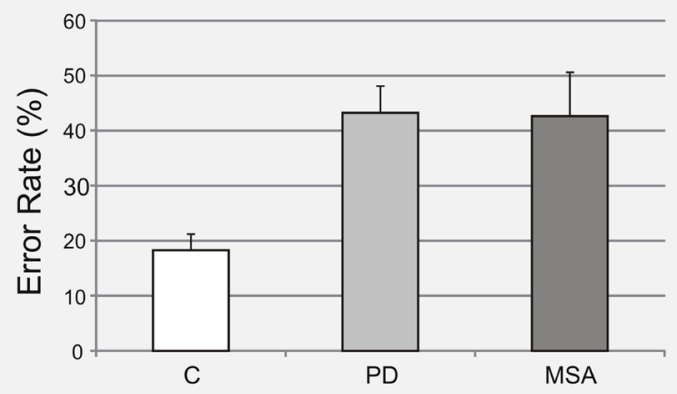

$\mathbf{F}$

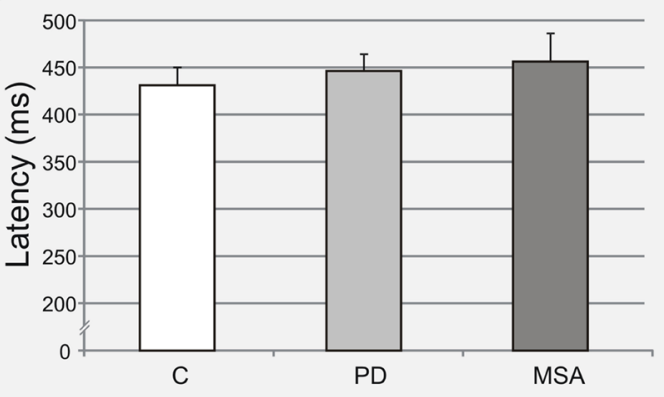

FIGURE 1 | Prosaccade and antisaccade tasks, error rates, and latencies. The prosaccade (A) and antisaccade (D) tasks are illustrated as a function of time. Error rates (unadjusted mean $\pm \mathrm{SE}$ ) for the prosaccade $\mathbf{( B )}$ and antisaccade (E) tasks are shown as percentages of the total number of trials for all groups. Latencies (unadjusted mean \pm SE) for the prosaccade (C) and antisaccade (F) tasks are shown for all groups. Control (C), Parkinson's disease (PD), and multiple system atrophy (MSA) subjects are illustrated in white, light gray, and dark gray, respectively.

$1.7 \%$ of remaining trials at $\mathrm{T} 1$, and 2.6 and $1.6 \%$ at $\mathrm{T} 2$, for prosaccade and antisaccade, respectively.

\section{Statistical Analyses}

For T1, saccade latencies were analyzed using mixed effect models and error rate was analyzed using generalized linear models with Poisson link for each task separately. The fixed effect was group (PD, MSA, control), and the random effect was the study subject. For group effects, we followed with post hoc planned comparisons among the three groups ( $\mathrm{C}$ vs PD; $\mathrm{C}$ vs MSA; PD vs MSA) to evaluate group differences and adjusted for covariates of age and gender and H\&Y score, when appropriate.

For longitudinal analyses examining progression, we performed mixed effect models for latencies and generalized linear models with Poisson link for error rates. The main factors in these models were group $(\mathrm{C}, \mathrm{PD}, \mathrm{MSA})$ and time point $(\mathrm{T} 1, \mathrm{~T} 2)$, and their interaction. We followed with post hoc planned within group comparisons (T2 vs T1) for each group (C, PD, MSA) to evaluate whether there were significant changes in performance (progression of disease or practice effects) across time points. We also performed post hoc planned comparisons of these changes (T1-T2) among the three groups (C vs PD; C vs MSA; PD vs MSA) to evaluate progression differences between groups. For all statistical tests, $p$-values $<0.05$ were considered statistically significant. Analyses were performed in SAS for Windows.

\section{Effect Size and Power}

A sample size of 11 participants is able to detect an effect size of $50 \mathrm{~ms}$ difference in RT between any two groups and a sample of 6 per group can detect a treatment effect of $42 \mathrm{~ms}$ in RT within a group at $80 \%$ power and $5 \%$ significance level. To account for difficulties in recruiting and repeated testing of elderly patients, 
we planned to recruit participants for each group until a total of six participants in both the PD and MSA groups were tested at T2.

\section{RESULTS}

Data were first summarized and reported as unadjusted means for T1 (Table 2) and for longitudinal analysis (Table 3). While there was no overall significant difference in age among groups, there were small but significant differences in age for some specific group comparisons. For consistency, the statistical effects and interactions reported below are adjusted for age.

\section{DIRECTION ERROR RATE}

\section{First Time Point, T1 \\ Prosaccades}

At $\mathrm{T} 1$, the direction error rate $( \pm \mathrm{SE})$ for prosaccade was $0.6 \%$ $( \pm 0.4), 1.5 \%( \pm 0.5)$, and $2.17 \%( \pm 1.0)$, for control, PD, and MSA groups, respectively (Table 2; Figure 1B). After adjusting for age effect $(p=0.0004)$, there was a significant difference among groups in prosaccade error rate $\left[\chi^{2}(2)=6.06, p=0.048\right]$. MSA participants made more errors than controls at $\mathrm{T} 1\left[\chi^{2}(1)=5.4\right.$, $p<0.02]$. MSA participants did not make more errors than PD participants $\left[\chi^{2}(1)=2.85, p=0.09\right]$, and there was no significant difference between PD and control groups in prosaccade error rate $\left[\chi^{2}(1)=1.0, p=0.31\right]$.

\section{Antisaccade}

The direction error rate $( \pm \mathrm{SE})$ for antisaccade task test at T1 was $18.2 \%( \pm 3.0 \%), 43.2 \%( \pm 4.9 \%)$, and $42.6 \%( \pm 8.0 \%)$, for control, PD, and MSA groups, respectively (Table 2; Figure 1E). After adjusting for age effect $(p=0.05)$, there was a significant difference among groups in error rate $\left[\chi^{2}(2)=86.26, p<0.001\right]$. Both PD and MSA participants made more errors than controls at $\mathrm{T} 1\left[\chi^{2}(1)=73.2\right.$, $p<0.001$ for PD versus controls; and $\chi^{2}(1)=45.6, p<0.001$ for MSA versus controls]. There was no significant difference between $\mathrm{PD}$ and MSA groups in antisaccade error rate $\left[\chi^{2}(1)=0.4, p=0.56\right]$.

\section{Longitudinal Comparisons, T1-T2 \\ Prosaccades}

Of the 20 subjects who were tested again at $\mathrm{T} 2$, the average prosaccade error rate changed from $0.4 \%( \pm 0.4 \%)$ to $1.7 \%( \pm 0.8 \%)$ for control group, $1.4 \%( \pm 0.8 \%)$ to $1.5 \%( \pm 0.8 \%)$ for PD group, and $1.5 \%( \pm 0.7 \%)$ to $2.7 \%( \pm 2.7 \%)$ for MSA group (Table 3$)$. There was no age effect $\left[\chi^{2}(1)=0.23, p=0.63\right]$ nor interaction effect between group and time point $\left[\chi^{2}(2)=1.09, p=0.58\right]$. In addition, there were no differences among groups $\left[\chi^{2}(2)=1.18\right.$, $p=0.55]$ or significant changes across time point collapsed across groups $\left[\chi^{2}(1)=1.57, p=0.21\right]$.

\section{Antisaccade}

The average antisaccade error rate changed from $16.2 \%( \pm 2.0)$ to $12.4 \%( \pm 2.5)$ for control group, $37.6 \%( \pm 7.8)$ to $34.8 \%( \pm 8.8)$ for PD group, and $37.8 \%( \pm 10.8)$ to $45.7 \%( \pm 12.8)$ for MSA group (Table 3; Figures 2A,B). Although the main effect of group was not significant $\left[\chi^{2}(2)=5.53, p=0.063\right]$, the paired comparison showed that PD and MSA participants had a 2.5- and 2.7-fold higher antisaccade error rates than controls, respectively $\left[\chi^{2}(1)=17.81, p<0.001\right.$ for PD versus controls; and $\chi^{2}(1)=17.07$, $p<0.001$ for MSA versus controls]. Additionally, time point was not significant $\left[\chi^{2}(1)=0.40, p=0.53\right]$. The change in antisaccade error rate (time point) was not significantly affected by age $\left[\chi^{2}(1)=0.00, p=0.98\right]$ nor by group [interaction between group and time point, $\chi^{2}(2)=2.55, p=0.28$; though PD and control participants improved, perhaps practice effects, whereas MSA participants made more errors].

\section{LATENCY}

\section{First Time Point, T1 Prosaccades}

At $\mathrm{T} 1$, the average prosaccade latency $( \pm \mathrm{SE})$ for correct trials was $260 \mathrm{~ms}( \pm 10)$ for control, $267 \mathrm{~ms}( \pm 8)$ for PD, and $318 \mathrm{~ms}$ $( \pm 20)$ for MSA group (Table 2; Figure 1C). After adjusting for age effects $(p<0.001)$, the mean latencies were different among groups $[F(2,48)=40.92, p<0.001]$. MSA patients were significantly slower than $\mathrm{PD}[t(48)=-8.18, p<0.001]$ and controls $[t(48)=-8.20, p<0.001]$, while there was no difference in mean latency between $\mathrm{PD}$ and controls $[t(51)=-0.21, p=0.84]$.

\section{Antisaccades}

In the antisaccade task, the average latency was $431 \mathrm{~ms}( \pm 19)$ for controls, $446 \mathrm{~ms}( \pm 18)$ for PD group, and $456 \mathrm{~ms}( \pm 30)$ for MSA group (Table 2; Figure 1F). After adjusting for age effect

TABLE 2 | Time point 1 unadjusted dependent measures for prosaccade and antisaccade tasks for control (C), Parkinson's disease (PD), and multiple system atrophy (MSA) groups.

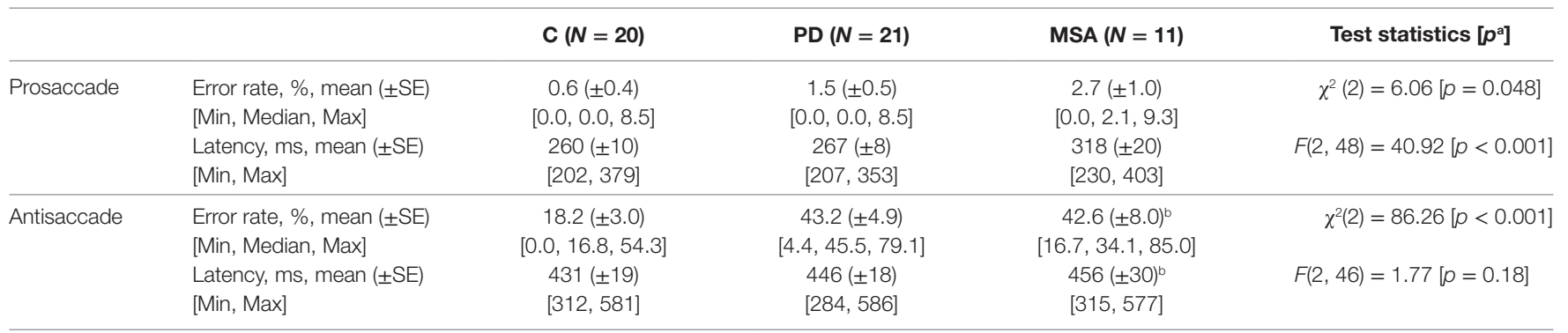

${ }^{a} p$ Values (p) for age adjusted group effects using generalized linear model with Poisson link for error rate and one way analysis of variance for latency. "As indicated in the Section "Data Analysis," 2 MSA subjects did not perform the antisaccade task ( $n=9$ for these means). 
$(p<0.001)$, the antisaccade latencies were not significantly different among groups $[F(2,46)=1.77, p=0.18]$.

\section{Longitudinal Comparisons, T1-T2 Prosaccade}

For participants who were retested, average prosaccade latency $( \pm$ SE) was $241 \mathrm{~ms}( \pm 12)$ at $\mathrm{T} 1$ and $235 \mathrm{~ms}( \pm 6)$ at T2 for controls, $247 \mathrm{~ms}( \pm 10)$ at $\mathrm{T} 1$ and $254 \mathrm{~ms}( \pm 9)$ at T2 for PD group, and $332 \mathrm{~ms}( \pm 34)$ at T1 and $308 \mathrm{~ms}( \pm 24)$ at T2 for MSA group (Table 3). After adjusting for age effect $(p=0.37)$, the change in latency between time points for each group, i.e., interaction between group and time point, was not significant $[F(2,31)=2.83$, $p=0.074]$. The main effect of time point was not significant $[F(1,31)=2.04, p=0.16]$. Consistent with $\mathrm{T} 1$, the group effect was significant $[F(2,31)=93.15, p<0.001]$, with the MSA group having the slowest prosaccade latencies.

\section{Antisaccades}

Average antisaccade latency $( \pm$ SE) changed from $409 \mathrm{~ms}( \pm 37)$ at T1 to $378 \mathrm{~ms}( \pm 38)$ at T2 for controls, $430 \mathrm{~ms}( \pm 31)$ to $407 \mathrm{~ms}$ $( \pm 27)$ for PD group, and $445 \mathrm{~ms}( \pm 38)$ to $440 \mathrm{~ms}( \pm 33)$ for MSA group (Table 3; Figures 2C,D). After adjusting for age effect $[F(1,33)=49.42, p<0.001]$, the changes in latency between time points among groups, interaction between group and time point, were similar $[F(2,32)=1.06, p=0.36]$. Overall, participants had a faster response time at $\mathrm{T} 2$ compared to $\mathrm{T} 1[F(1,33)=5.92$, $p=0.021]$; however, the MSA patients were not faster at T2 compared to $\mathrm{T} 1[t(33)=0.13, p=0.90]$. Unlike the T1 analysis, the group effect was significant $[F(2,33)=10.97, p<0.001]$, with the MSA group having the slowest latencies at T2 $[t(33)=-4.13$, $p<0.001$ for control versus MSA; and $t(33)=3.19, p=0.003$ for $\mathrm{PD}$ versus $\mathrm{MSA}]$.

\section{CONTROLLING FOR AGE AND DISEASE SEVERITY ACROSS PATIENT GROUPS}

Given prior work showing that age (31) and disease severity $(30,32)$ can affect eye movement measures, we reanalyze the data from the two patient groups (PD and MSA), controlling for age and $\mathrm{H} \& \mathrm{Y}$ score together.

\section{Direction Error Rate}

For prosaccades, as reported above, there was no significant difference between the two groups. After controlling for age and $\mathrm{H} \& \mathrm{Y}$ score together, there remained no significant differences between the two groups $\left[\chi^{2}(1)=1.35, p=0.25\right]$. For antisaccades, as reported above, there was no significant difference between the two groups, even when controlling for a significant effect of age. After controlling for age and $\mathrm{H} \& \mathrm{Y}$ score together, there again was no significant difference between the two groups $\left[\chi^{2}(1)=1.35\right.$, $p=0.25]$.

\section{Latency}

For prosaccades, as reported above, there was a significant difference between the two groups (MSA slower than PD). After controlling for age and $\mathrm{H} \& \mathrm{Y}$ score together, there remained a significant difference between the two groups $[t(28)=-3.65$, 


\section{A}

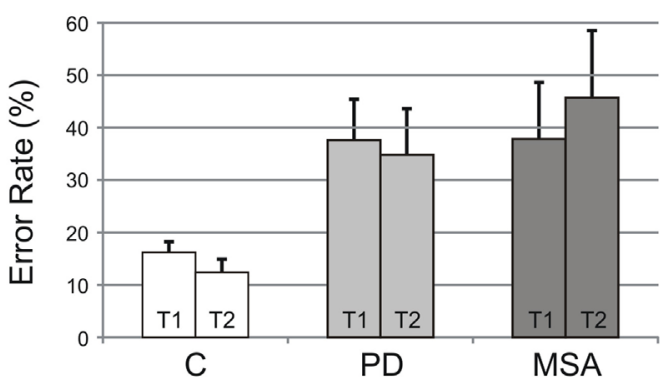

C

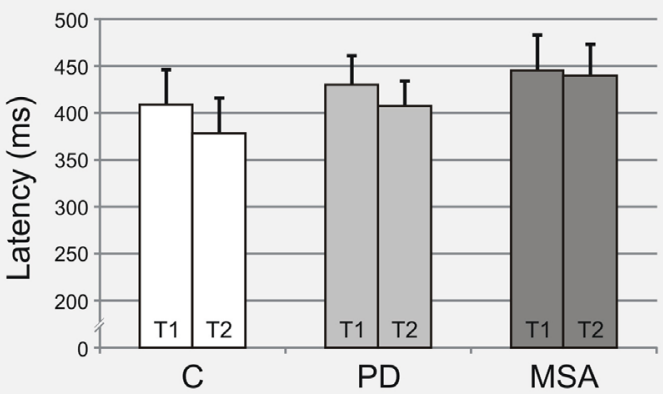

B

Change In Performance (T1-T2)

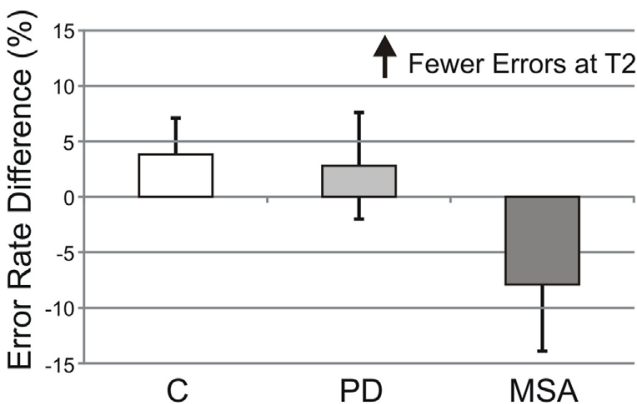

D

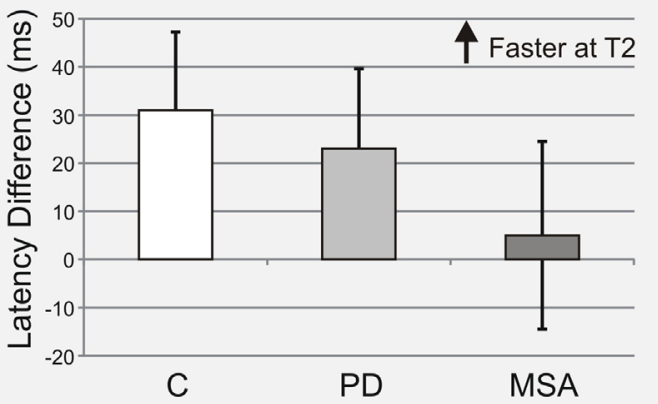

FIGURE 2 | Longitudinal data indicate improved performance over time. (A) Antisaccade error rates for time point 1 (T1) and time point 2 (T2) are shown for each group tested (unadjusted mean \pm SE): Control subjects (C), Parkinson's disease (PD), and multiple system atrophy (MSA). (B) Differences in antisaccade error rates (T1-T2), with fewer errors at T2 indicated by positive values, for all groups. (C) Antisaccade latencies for time point 1 (T1) and time point 2 (T2) are shown for each group tested (unadjusted mean \pm SE): control subjects (C), PD, and multiple system atrophy (MSA). (D) Differences in antisaccade latencies (T1-T2), with shorter latencies at T2 indicated by positive values, for all groups. Group bar coloring in all panels is the same as in Figure $\mathbf{1 .}$

$p=0.001]$. Thus, when controlling for both age and H\&Y score, MSA participants were on average $29 \mathrm{~ms}$ slower than PD participants. For antisaccades, as reported above, there was no significant difference between the groups, even when controlling for a significant effect of age. After controlling for age and H\&Y score together, there again was no significant difference between the two groups $[t(28)=0.42, p=0.68]$.

\section{DISCUSSION}

At a single time point, the study revealed two important findings: (1) error rates of MSA and PD patients in the antisaccade task were higher than controls, suggesting a behavioral biomarker for risk of a Parkinsonian disorder and (2) MSA patients show slower prosaccade latency than both controls and PD patients, suggesting a useful early behavioral marker for distinguishing MSA from PD. In addition, across a relatively short time frame ( $\sim$ months), repeated testing suggested antisaccade performance might also distinguish MSA from PD and may be a sensitive measure of the progression of cognitive deficit in MSA.

\section{High Error Rates}

High antisaccade error rates of PD and MSA reflect the common frontal brain deficits characteristic of both groups. These deficits (e.g., disruption of dopaminergic input to the prefrontal-basal ganglia circuitry) result in impaired inhibition of impulsive or reflexive responses and impaired generation of voluntary movements. With difficulties suppressing reflexive responses as well as with initiating correct voluntary saccades, the observed high error rates in PD and MSA compared to controls may be a useful behavioral biomarker to distinguish normal aging from Parkinsonian syndromes.

Much previous work suggests voluntary saccade deficits in $\mathrm{PD}(13,22,35)$, and recent work is consistent with this finding of similar voluntary saccade deficits using a different voluntary eye movement paradigm. For example, in a memory guided saccade paradigm, Terao and colleagues (30) demonstrated a deficit in voluntary control (i.e., increased saccades to cue or inability to suppress reflexive saccades during a memory-guided saccade paradigm) in MSA-P patients with no significant differences between PD and MSA-P patients. Also, in our study, there was some indication that MSA but not PD patients show increased prosaccade errors compared to controls. Perhaps consistent with this finding, recent work has demonstrated MSA-P patients make hypometric saccades compared to a normal age-matched control group (28) and that visually guided saccade accuracy (amplitude) was significantly reduced in MSA-P patients relative to PD patients (36). Further research is necessary to determine if higher error rates onset differentially in PD and MSA. 


\section{Prosaccade Slowing}

The significantly slower reflexive latencies of MSA patients likely reflect the underlying differences in MSA versus PD pathology. Specifically, impairments to visual cortex and brainstem regions in MSA could result in more impaired reflexive oculomotor function. Previous work demonstrated slower reflexive latencies of both MSA-P and PD patients and suggested equivalent slowing (36). Although the study was well controlled for clinical severity, it differed from the current study in that the PD group was not in the conventionally defined "off" state (i.e., more than $12 \mathrm{~h}$ without dopaminergic medication). Thus, it is possible that there were no differences between PD and MSA in that study due to dopaminergic drug load (24). The normal reflexive latencies of the PD patients off medication in our study suggest that these patients experience less widespread degeneration at this stage. Specifically, MSA patients may differ from PD in that they undergo early decline in reflexive function as well as early frontal lobe dysfunction. Together, high antisaccade error rates and prosaccade slowing may be a useful marker for early differentiation between PD and MSA.

\section{Disease Progression and Severity}

From the longitudinal data, there was no significant group by time point interactions for any of the dependent measures, suggesting that no significant changes occur over a short interval ( $\sim$ months) that could be used to distinguish between MSA and PD. Although the group-time point interactions were non-significant, there was an additional group effect that emerged in antisaccade latency. Overall, participants had shorter latencies in the repeated testing sessions; however, while C and PD showed greater benefits (shorter latencies) during retesting, the MSA group did not (Figures 2C,D). These group differences could be interpreted as a reduced cognitive benefit in MSA patients with repeated testing due to impaired learning, or more rapid and widespread disease progression, or a combination of both. Interestingly, a similar but non-significant interaction pattern supportive of these latency changes was also present in antisaccade error rates (Figures $\mathbf{2 A , B}$ ): small performance benefits across time points in $\mathrm{C}$ and $\mathrm{PD}$, but a small performance decrement in MSA, perhaps suggestive of impaired learning and progression. Additional work is needed to establish such group differences in performance across time and to distinguish the cause of any putative changes.

Given the faster progression of disease in MSA patients, in our mid-stage patients, there was a significant difference in clinical severity, as measured by the H\&Y. Thus, it is possible that differences in clinical severity were the cause of slower prosaccade latencies in our MSA group. Terao and colleagues have shown that there are changes in PD eye movement measures with disease progression (30). However, saccade parameters are also known to be affected by both age (37) and dopaminergic medication (24), which remain potential confounds in their study. Terao et al. (36) also showed that some saccade parameters in MSA-P patients vary with disease progression, but again, age and dopaminergic medication load (only after $4 \mathrm{~h}$ ) could have affected their findings. In the present study, we tested patients in the "off" state and when we controlled for both age and H\&Y, our main findings regarding elevated AS errors in both groups and slower prosaccades in MSA patients were not affected.

\section{Limitations and Future Directions}

These biomarkers may only be useful for early-to-moderate PD patients. Prospective investigations at an earlier time point in the disease course are critical to determine whether the observed changes in dependent measures can differentiate PD and MSA groups before definitive clinical diagnosis. Additionally, it is important to note that our MSA group is composed almost equally of MSA-P and MSA-C patients, which differs from the phenotype that is predominant in countries like Japan (MSA-C). Future investigations using homogeneous subtype populations, more time points, or a longer time interval would be helpful to discern group differences in the progression of disease.

\section{Health Relevance}

As new neuroprotective agents are developed, early and accurate diagnosis of Parkinsonian disorders becomes paramount. Our study indicates that increased antisaccade errors combined with slowed prosaccade latencies might serve as a behavioral biomarker for early differentiation between PD and MSA. Additionally, changes in antisaccade performance may prove useful for tracking MSA disease progression and evaluating potential interventions.

\section{ETHICS STATEMENT}

Subjects provided written informed consent in accordance with the Declaration of Helsinki and were enrolled into a study approved by the Committee for the Protection of Human Subjects, the Institutional Review Board at the University of Texas Health Science Center at Houston.

\section{AUTHOR CONTRIBUTIONS}

SB: data collection, analysis, and manuscript preparation. EK: data collection, analysis, and manuscript preparation and review. SR: data collection and initial analysis. NM: data collection and manuscript review. SP: data analysis, manuscript preparation, and review. AC: statistical analysis, interpretation, manuscript preparation, and review. JS: clinical testing, clinical data collection, manuscript preparation, and review. MS: subject recruitment, clinical testing, evaluation and diagnoses, and manuscript review. AS: study formulation, design, data collection and analysis, and manuscript preparation. All authors: contributed by drafting parts of the work, have approved the final version, and agreed to be accountable for all aspects of the work.

\section{ACKNOWLEDGMENTS}

The authors would like to thank Vicki Ephron for participant care and facilitation between laboratory and clinical settings.

\section{FUNDING}

This work was supported by Mission Connect, a program of TIRR Foundation (grant number 014-119); a Catalyst Pilot Award; and by the National Institutes of Health (grant number P30-EY010608). 


\section{REFERENCES}

1. Williams DR, Litvan I. Parkinsonian syndromes. Continuum (2013) 19: 189-212. doi:10.1212/01.CON.0000436152.24038.e0

2. Wenning GK, Litvan I, Tolosa E. Milestones in atypical and secondary parkinsonisms. Mov Disord (2011) 26:1083-95. doi:10.1002/mds.23713

3. Brooks DJ. Diagnosis and management of atypical parkinsonian syndromes. J Neurol Neurosurg Psychiatry (2002) 72:110-16. doi:10.1136/ jnnp.72.suppl_1.i10

4. Hughes AJ, Daniel SE, Kilford L, Lees AJ. Accuracy of clinical diagnosis of idiopathic Parkinson's disease: a clinico-pathological study of 100 cases. J Neurol Neurosurg Psychiatry (1992) 55:181-4. doi:10.1136/jnnp. 55.3.181

5. Schrag A, Good CD, Miszkiel K, Morris HR, Mathias CJ, Lees AJ, et al. Differentiation of atypical parkinsonian syndromes with routing MRI. Neurology (2000) 54:697-702. doi:10.1212/WNL.54.3.697

6. Lantos PL. The definition of multiple system atrophy: a review of recent developments. J Neuropathol Exp Neurol (1998) 57:1099-111. doi:10.1097/ 00005072-199812000-00001

7. Köllensperger M, Geser F, Ndayisaba JP, Boesch S, Seppi K, Ostergaard K, et al. Presentation, diagnosis, and management of multiple system atrophy in Europe: final analysis of the European multiple system atrophy registry. Mov Disord (2010) 25:2604-12. doi:10.1002/mds.23192

8. Gilman S, Wenning GK, Low PA, Brooks DJ, Mathias CJ, Trojanowski JQ, et al. Second consensus statement on the diagnosis of multiple system atrophy. Neurology (2008) 71:670-6. doi:10.1212/01.wnl.0000324625.00404.15

9. White OB, Fielding J. Cognition and eye movements: assessment of cerebral dysfunction. J Neuroophthalmol (2012) 32:266-73. doi:10.1097/ WNO.0b013e3182688230

10. Anderson TJ, MacAskill MR. Eye movements inpatients with neurodegenerative disorders. Nat Rev Neurol (2013) 9:74-85. doi:10.1038/nrneurol.2012.273

11. Van Stockum S, MacAskill MR, Myall D, Anderson TJ. A perceptual discrimination task abnormally facilitates reflexive saccades in Parkinson's disease. Eur J Neurosci (2011) 33:2091-100. doi:10.1111/j.1460-9568.2011.07697.x

12. Van Stockum S, MacAskill MR, Myall D, Anderson TJ. A perceptual discrimination task results in greater facilitation of voluntary saccades in Parkinson's disease patients. Eur J Neurosci (2013) 37:163-72. doi:10.1111/ejn.12033

13. Vidailhet M, Rivaud S, Gouider-Khouja N, Pillon B, Bonnet AM, Gaymard B, et al. Eye movements in parkinsonian syndromes. Ann Neurol (1994) 35:420-6. doi:10.1002/ana.410350408

14. Guitton D, Buchtel HA, Douglas RM. Frontal lobe lesions in man cause difficulties in suppressing reflexive glances and in generating goal-directed saccades. Exp Brain Res (1985) 58:455-72. doi:10.1007/BF00235863

15. Munoz DP, Dorris MC, Pare M, Everling S. On your mark, get set: brainstem circuitry underlying saccadic initiation. Can J Physiol Pharmacol (2000) 78:934-44. doi:10.1139/y00-062

16. Munoz DP, Everling S. Look away: the anti-saccade task and the voluntary control of eye movement. Nat Rev Neurosci (2004) 5:218-28. doi:10.1038/ nrn 1345

17. Schiller PH, Sandell JH, Maunsell JH. The effect of frontal eye field and superior colliculus lesions on saccadic latencies in the rhesus monkey. J Neurophysiol (1987) 57:1033-49.

18. Ettinger U, Kumari V, Chitnis XA, Corr PJ, Crawford TJ, Fannon DG, et al. Volumetric neural correlates of antisaccade eye movements in first-episode psychosis. Am J Psychiatry (2004) 161:1918-21. doi:10.1176/ ajp.161.10.1918

19. Ford KA, Goltz HC, Brown MR, Everling S. Neural processes associated with antisaccade task performance investigated with event-related FMRI. J Neurophysiol (2005) 94:429-40. doi:10.1152/jn.00471.2004

20. Miller LM, Sun FT, Curtis CE, D'Esposito M. Functional interactions between oculomotor regions during prosaccades and antisaccades. Hum Brain Mapp (2005) 26:119-27. doi:10.1002/hbm.20146

21. Wang CA, Brien DC, Munoz DP. Pupil size reveals preparatory processes in the generation of pro-saccades and anti-saccades. Eur J Neurosci (2015) 41:1102-10. doi:10.1111/ejn.12883
22. Briand KA, Strallow D, Hening W, Poizner H, Sereno AB. Control of voluntary and reflexive saccades in Parkinson's disease. Exp Brain Res (1999) 129:38-48. doi:10.1007/s002210050934

23. Gooding DC, Basso MA. The tell-tale tasks: a review of saccadic research in psychiatric patient populations. Brain Cogn (2008) 68:371-90. doi:10.1016/j. bandc.2008.08.024

24. Hood AJ, Amador SC, Cain AE, Briand KA, Al-Refai AH, Schiess MC, et al. Levodopa slows prosaccades and improves antisaccades: an eye movement study in Parkinson's disease. J Neurol Neurosurg Psychiatry (2007) 78:565-70. doi:10.1136/jnnp.2006.099754

25. Peltsch A, Hemraj A, Garcia A, Munoz DP. Saccade deficits in amnesic mild cognitive impairment resemble mild Alzheimer's disease. Eur JNeurosci (2014) 39:2000-13. doi:10.1111/ejn.12617

26. Pierrot-Deseilligny C, Rivaud S, Gaymard B, Agid Y. Cortical control of reflexive visually-guided saccades. Brain (1991) 114:1473-85. doi:10.1093/ brain/114.3.1473

27. Anderson T, Luxon L, Quinn N, Daniel S, Marsden CD, Bronstein A. Oculomotor function in multiple system atrophy: clinical and laboratory features in 30 patients. Mov Disord (2008) 23:977-84. doi:10.1002/mds.21999

28. Terao Y, Fukuda H, Tokushige SI, Inomata-Terada S, Yugeta A, Hamada M, et al. Distinguishing spinocerebellar ataxia with pure cerebellar manifestation from multiple system atrophy (MSA-C) through saccade profiles. Clin Neurophysiol (2017) 128:31-43. doi:10.1016/j.clinph.2016.10.012

29. Chambers JM, Prescott TJ. Response times for visually guided saccades in persons with Parkinson's disease: a meta-analytic review. Neuropsychologia (2010) 48:887-99. doi:10.1016/j.neuropsychologia.2009.11.006

30. Terao Y, Fukuda H, Yugeta A, Hikosaka O, Nomura Y, Segawa M, et al. Initiation and inhibitory control of saccades with the progression of Parkinson's disease - changes in three major drives converging on the superior colliculus. Neuropsychologia (2011) 49:1794-806. doi:10.1016/j. neuropsychologia.2011.03.002

31. Irving EL, Steinbach MJ, Lillakas L, Babu RJ, Hutchings N. Horizontal saccade dynamics across the human life span. Invest Ophthalmol Vis Sci (2006) 47:2478-84. doi:10.1167/iovs.05-1311

32. Patel SS, Jankovic J, Hood AJ, Jeter CB, Sereno AB. Reflexive and volitional saccades: biomarkers of Huntington disease severity and progression. J Neurol Sci (2012) 313:35-41. doi:10.1016/j.jns.2011.09.035

33. Goetz CG, Fahn S, Martinez-Martin P, Poewe W, Sampaio C, Stebbins GT, et al. Movement Disorder Society-sponsored revision of the Unified Parkinson's Disease Rating Scale (MDS-UPDRS): Process, format, and clinimetric testing plan. Mov Disord (2007) 22:41-7. doi:10.1002/mds.21198

34. Hoehn MM, Yahr MD. Parkinsonism: onset, progression, and mortality. Neurology (1967) 17:427-42. doi:10.1212/WNL.17.5.427

35. Bronstein AM, Kennard C. Predictive ocular motor control in Parkinson's disease. Brain (1985) 108:925-40. doi:10.1093/brain/108.4.925

36. Terao Y, Fukuda H, Tokushige S, Inomata-Terada S, Yugeta A, Hamada M, et al. Is multiple system atrophy with cerebellar ataxia (MSA-C) like spinocerebellar ataxia and multiple system atrophy with parkinsonism (MSA-P) like Parkinson's disease? - A saccade study on pathophysiology. Clin Neurophysiol (2016) 127:1491-502. doi:10.1016/j.clinph.2015.07.035

37. Munoz DP, Broughton JR, Goldring JE, Armstrong IT. Age-related performance of human subjects on saccadic eye movement tasks. Exp Brain Res (1998) 121:391-400. doi:10.1007/s002210050473

Conflict of Interest Statement: The authors declare that the research was conducted in the absence of any commercial or financial relationship that could be construed as a potential conflict of interest.

Copyright (C) 2017 Brooks, Klier, Red, Mehta, Patel, Chuang, Suescun, Schiess and Sereno. This is an open-access article distributed under the terms of the Creative Commons Attribution License (CC BY). The use, distribution or reproduction in other forums is permitted, provided the original author(s) or licensor are credited and that the original publication in this journal is cited, in accordance with accepted academic practice. No use, distribution or reproduction is permitted which does not comply with these terms. 